\title{
How anisotropic and isotropic atomic displacement parameters monitor protein covalent bonds rigidity: isotropic B-factors underestimate bond rigidity
}

\author{
Oliviero Carugo ${ }^{1,2}$ (B)
}

Received: 24 November 2020 / Accepted: 21 April 2021 / Published online: 29 April 2021

(c) The Author(s) 2021

\begin{abstract}
Under the assumption that covalent bonds are rigid, it is possible to compare the estimations of rigidity based on anisotropic and isotropic B-factors. This is done by computing the difference of the mean-square displacements (Delta-u) of atoms A and $\mathrm{Z}$ along the covalent bond $\mathrm{A}-\mathrm{Z}$, which must be close to zero for a rigid bond. The analysis of a high-quality set of protein structures, refined at a resolution better than (or equal to) 0.8 Angstroms, showed that Delta-u is significantly close to zero when anisotropic B-factors are used, with an average 60\% Delta-u reduction. This reduction is larger for larger B-factors and this suggests that care should be taken in data-mining procedures that involve isotropic B-factors, especially at lower resolution, when anisotropic B-factors cannot be determined and when the average B-factor increases.
\end{abstract}

Keywords Atomic displacement parameter $\cdot$ Atomic resolution $\cdot$ B-factor $\cdot$ Covalent bond $\cdot$ Hirshfeld rigidity test $\cdot$ Protein structure

The benefits of high-resolution macromolecular crystal structures have been repeatedly described (Longhi et al. 1998; Dauter et al. 1997; Schmidt and Lamzin 2002; Schmidt and Lamzin 2010). The wealth of details in electron density maps at high resolution allows the characterization of the conformational disorder of many protein atoms, which may show two or three stable positions, and of the protein hydration by water molecules that cover the protein surface (Schmidt and Lamzin 2010; Schmidt et al. 2011; Bhattacharjee and Biswas 2011). A remarkable feature of highresolution crystal structures is the anisotropic refinement of the atomic displacement parameters (therein after referred to as B-factors), which is impossible at lower resolution and which provides valuable information on local dynamics (Schmidt et al. 2011; Carugo 2020; Carugo 2019a). However, a systematic comparison—based on independent and external criteria - of anisotropic B-factors, which can

Oliviero Carugo

Oliviero.carugo@univie.ac.at

1 Department of Chemistry, University of Pavia, Viale Taramelli 12, 27100 Pavia, Italy

2 Department of Structural and Computational Biology, University of Vienna, Vienna, Austria be refined only at high resolution, and isotropic B-factors, which are routinely refined at lower resolution, have never been published.

Anisotropic and isotropic B-factors can be compared by analyzing their ability to monitor the rigidity of the protein covalent bonds, a feature that is independent of the refinement level but is directly related to the electronic structure of the proteins.

Covalent bonds are highly rigid and their deformation implies severe energy costs (Slater 1968). This has been exploited in macromolecular crystallography with the introduction of refinement restraints (Tronrud 1996; Thorn et al. 2012; Parois et al. 2018), which essentially assume that the difference of the mean-square displacements of atoms $A$ and $\mathrm{Z}$ along the covalent bond $\mathrm{A}-\mathrm{Z}$ must be close to zero. These restraints are usually relaxed at very high resolution, when they are no more essential to ensure a physically and chemically realistic structure description.

Axiomatically, it is possible to assume that covalent bond is rigid, especially at low temperature-crystal structures are routinely determined at $100 \mathrm{~K}$ nowadays - and this is monitored by the Hirschfeld test (Hirshfeld 1976), according to which, as mentioned above, the components of the B-factors of the two atoms along the covalent bond must be the same. 
When isotropic B-factors (B) are available, the meansquare displacement $(\mathrm{u})$ of an atom is the same in all directions around the atomic average position, and the rigidity of the bond $\mathrm{A}-\mathrm{Z}$ can be monitored by the function Delta- $u$, defined as:

Delta $-u=\left|u_{A}-u_{Z}\right|=\left|\sqrt{\frac{B_{A}}{8 \pi^{2}}}-\sqrt{\frac{B_{Z}}{8 \pi^{2}}}\right|$,

which must be close to $0 \AA$, according to the Hirshfeld rigidity test (Hirshfeld 1976) $\left(B_{A}\right.$ and $B_{Z}$ are the B-factors of atoms $\mathrm{A}$ and $\mathrm{Z}$ ). With anisotropic B-factors $(\mathbf{U})$, the Delta- $u$ function must consider that the mean-square displacement (u) of an atom is not the same in all directions, and it must be computed as:

Delta $-u=\left|n^{\mathrm{T}} \mathrm{U}_{\mathrm{A}} n-n^{\mathrm{T}} \mathrm{U}_{\mathrm{Z}} n\right|$,

where $n$ is the unit vector in the covalent bond direction, and $\boldsymbol{U}_{\boldsymbol{A}}$ and $\boldsymbol{U}_{\boldsymbol{Z}}$ are the anisotropic B-factors of atoms $\mathrm{A}$ and Z (Burgi 1994).

In the present communication, isotropic and anisotropic Delta- $u$ values were computed and compared in a nonredundant set of extremely high-resolution protein crystal structures extracted from the Protein Data Bank (Bernstein et al. 1977; Berman et al. 2000).

Only X-ray crystal structures refined at a resolution of at least $0.8 \AA$ and determined in the $90-100 \mathrm{~K}$ temperature range were retained, and the following thirty crystal structures were eventually kept-chain identifiers in parentheses-once the sequence redundancy was reduced to $40 \%$ pairwise sequence identity with CD-HIT ( $\mathrm{Li}$ and Godzik 2006; Fu et al. 2012): 1ejg(A), 1 gci(A), 1iua(A), 1r6j(A), $1 \mathrm{ucs}(\mathrm{A}), 1 \mathrm{us} 0(\mathrm{~A}), 1 \mathrm{w} 0 \mathrm{n}(\mathrm{A}), 1 \times 6 \mathrm{z}(\mathrm{A}), 2 \mathrm{~b} 97(\mathrm{~A}), 2 \mathrm{ixt}(\mathrm{A})$, 2izq(A), 2ov0(A), 2pve(A), 2vb1(A), 2wfi(A), 3mfj(A), 3ui4(A), $3 \times 2 \mathrm{~m}(\mathrm{~A}), 3 \times 34(\mathrm{~A}), 4 \mathrm{hp} 2(\mathrm{~A}), 4 \operatorname{rek}(\mathrm{A}), 4 \mathrm{ua} 6(\mathrm{~A})$, $5 \mathrm{al6}(\mathrm{A}), 5 \mathrm{kwm}(\mathrm{A}), 5 \mathrm{nfm}(\mathrm{A}), 5 \mathrm{tda}(\mathrm{A}), 5 \mathrm{yce}(\mathrm{A}), 6 \mathrm{e} 6 \mathrm{o}(\mathrm{A})$, 6127(A), 6s2m(A).

Solvent accessible surface areas for each atom were computed with Naccess (Hubbard and Thornton 1993) and all the other computations were performed with locally written software.

Anisotropic Delta- $u$ was computed for all protein covalent bonds with anisotropic B-factors (Eq. 1), and isotropic Delta- $u$ was computed with the equivalent isotropic B-factors (Eq. 2), which, for anisotropically refined atoms, are equal to

$B=8 \pi^{2} \frac{U_{11}+U_{22}+U_{33}}{3}$

In general, and as expected, anisotropic Delta- $u$ is smaller than isotropic Delta- $u$ for all types of covalent bonds (see an example in Figure S1, Supplementary Material): on average, it is $0.0108(0.0001) \AA$ smaller. This difference is larger for side-chain bonds [0.0157 (0.0002) $\AA$ ] than for main-chain bond [0.0061 (0.0002) $\AA$ ]. Only for $13 \%$ of the bonds, the isotropic Delta- $u$ is slightly smaller than the anisotropic Delta- $u$ and most of these cases concern the $\mathrm{C}-\mathrm{N}$ backbone bonds. This percentage is smaller for side-chain bonds (10\%) than for backbone bonds (17\%).

This is certainly not surprising. In fact, given the assumption that covalent bonds are rigid, this reflects the better modeling of atomic dispersion around the equilibrium positions in anisotropic refinements. In other words, the rigidity of covalent bonds is better accounted for by anisotropic B-factors refinements, especially for side-chains, which tend to be more flexible.

However, the added value of the work presented here is the fact that this is a quantitative comparison, which points out that, given that the anisotropic and isotropic Delta- $u$ are equal, on average, to 0.0075 (0.0001) and 0.0184 (0.0001) $\AA$, the deviation from rigidity of the covalent bonds is reduced by $60 \%$ when anisotropic B-factors are refined. This improvement is remarkable and somehow surprising by its amount.

This can be appreciated also by the relationship between the anisotropic and isotropic Delta- $u$ shown in Fig. 1a. While the isotropic Delta- $u$ increases considerably, from values close to $0 \AA$ up to $0.04 \AA$, the anisotropic Delta- $u$ increases much less, only from 0.006 to $0.007 \AA$ to about $0.01 \AA$. This confirms that the covalent bond rigidity is drastically better monitored by anisotropic B-factors.

Interestingly, both anisotropic and isotropic Delta- $u$ tend to be larger for atom pairs that have a larger average isotropic B-factor (Fig. 1b). However, this is more pronounced for isotropic Delta- $u$, which increases from 0.01 to $0.04 \AA$ when B increases from 5 to $20 \AA^{2}$-note that the latter value is close to the maximum possible $\mathrm{B}$ value at very high resolution (Carugo 2018a, 2019b). On the contrary, the anisotropic Delta- $u$ increases much less in the same B-factor range. This is represented, in the figure, by the fact that also the difference between isotropic and anisotropic Delta- $u$ increases if the B-factors increase. This clearly indicates that large atomic positional dispersions cannot be described effectively by isotropic B-factor.

Similar trends are observed when the average solvent accessible surface area of the atoms that are covalently bound is considered (Fig. 1c). The isotropic Delta- $u$ values increase if the solvent accessible area surface area increases, indicating that the rigidity is larger for covalent bonds buried in the protein core than for covalent bonds exposed to the solvent. On the contrary, the anisotropic Delta- $u$ is nearly constant in the examined range of solvent accessible surface area and, as a consequence, the difference between isotropic and anisotropic Delta- $u$ increases if the solvent accessibility increases. This supports the previous observations on 

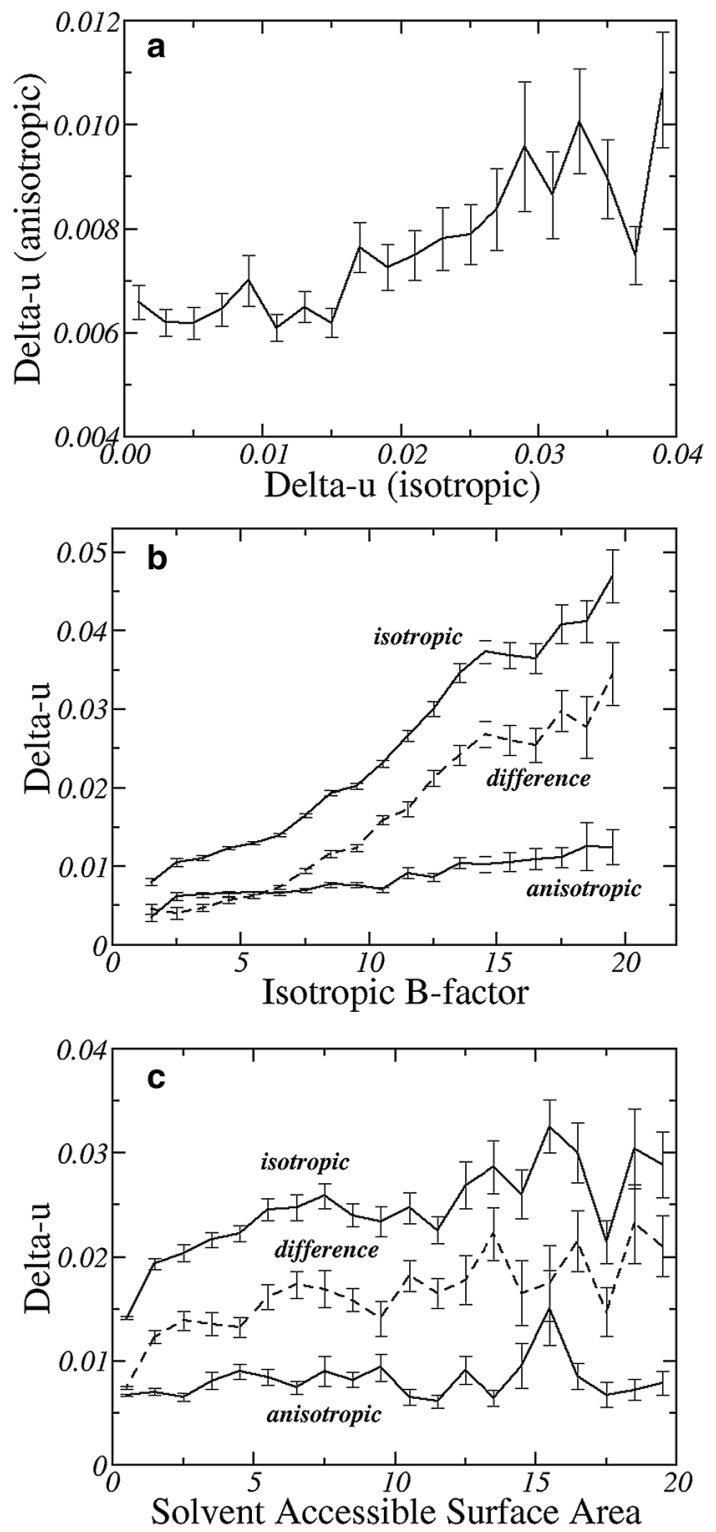

Fig. 1 Relationship between isotropic and anisotropic Delta-u ( $\AA$, a); between the Delta-u $(\AA)$ and the average isotropic B-factor of the two atoms that are covalently bound $\left(\AA^{2} ; \mathbf{b}\right)$; and between the Delta-u $(\AA)$ and the average solvent accessible surface area of the two atoms that are covalently bound $\left(\AA^{2} ; \mathbf{c}\right)$. Both backbone and side-chain bonds, which are equally numerous, were considered

the relationships between Delta- $u$ and equivalent isotropic $\mathrm{B}$-factor and is not surprising since larger isotropic B-factors are expected for atoms more exposed to the solvent.

The data available in the Protein Data Bank allow one to estimate that the covalent bond rigidity is much better accounted for by anisotropic B-factors than by isotropic B-factors. A remarkable $60 \%$ reduction of the deviation from rigidity is observed, on average. If on the one side this is expected, on the other side, it points out that the information provided by isotropic B-factors is of limited accuracy when protein dynamics must be quantitatively evaluated at a molecular level. Care should then be taken in data-mining procedures that involve isotropic B-factors (Carugo 2018b; Sun, Qu, Feng, Reetz 2019), from drug design (Johnson et al. 2018), to atom position accuracy estimation (Dinesh Kumar et al. 2015), or to protein engineering (Reetz et al. 2006).

It is also important to remember that the inaccuracies of isotropic B-factors estimated in the present communication might be underestimated, since isotropic Delta- $u$ was computed with isotropic equivalent B-factors and not with genuine isotropic B-factors. In other words, isotropic Delta$u$ was computed with isotropic B-factors that resulted from anisotropic refinements and not with isotropic B-factors that can be refined at lower resolution.

Supplementary Information The online version contains supplementary material available at https://doi.org/10.1007/s00726-021-02985-x.

Acknowledgements I express my gratitude to Kristina DjinovićCarugo for helpful discussions and hospitality in her lab and to Gottlieb Muffat for constant support.

Funding Open access funding provided by University of Vienna. No funding was necessary and received for this study.

\section{Declarations}

Conflict of interest The author has no conflict of interest.

Ethical approval No human participants or animals were used in this study. An informed consent declaration is, therefore, not necessary.

Open Access This article is licensed under a Creative Commons Attribution 4.0 International License, which permits use, sharing, adaptation, distribution and reproduction in any medium or format, as long as you give appropriate credit to the original author(s) and the source, provide a link to the Creative Commons licence, and indicate if changes were made. The images or other third party material in this article are included in the article's Creative Commons licence, unless indicated otherwise in a credit line to the material. If material is not included in the article's Creative Commons licence and your intended use is not permitted by statutory regulation or exceeds the permitted use, you will need to obtain permission directly from the copyright holder. To view a copy of this licence, visit http://creativecommons.org/licenses/by/4.0/.

\section{References}

Berman HM, Westbrook J, Feng Z, Gilliland G, Bhat TN, Weissig H, Shindyalov IN, Bourne PE (2000) The Protein Data Bank. Nucleic Acids Res [Internet] 28:235-242

Bernstein FC, Koetzle TF, Williams GJB, Meyer EFJ, Brice MD, Rodgers JR, Kennard O, Shimanouchi T, Tasumi M (1977) The Protein Data Bank: a computer-based archival file for macromolecular structures. J Mol Biol 112:535-542

Bhattacharjee N, Biswas P (2011) Structure of hydration water in proteins: A comparison of molecular dynamics simulations and database analysis. Biophys Chem 158:73-80 
Burgi HB, Dunitz JD (1994) Structure correlation. (Buergi HB, editor.). Weinheim: Verlagsgesellschaft $\mathrm{mbH} ; 1994$

Carugo O (2018a) How large B-factors can be in protein crystal structures. BMC Bioinformatics 19:61

Carugo O (2018b) Atomic displacement parameters in structural biology. Amino Acids [Internet] 50:775-786

Carugo O (2019a) Anisotropic waters in atomic resolution protein crystal structures. Int J Biol Macromol 135:940-944

Carugo O (2019b) Maximal B-factors in protein crystal structures. Zeit Krist 234:73-77

Carugo O (2020) Mobility of water and of protein atoms at the proteinwater interface, monitored by anisotropic atomic displacement parameters, are largely uncorrelated. Amino Acids 52:435-443

Dauter Z, Lamzin VS, Wilson KS (1997) The benefits of atomic resolution. Curr Opin Struct Biol 7:681-688

Dinesh Kumar KS, Gurusaran M, Satheesh SN, Radha P, Pavithra S, Thulaa Tharshan KPS, Helliwell JR, Sekar K (2015) Online_DPI: a web server to calculate the diffraction precision index for a protein structure. J Appl Cryst 48:939-942

Fu L, Niu B, Zhu Z, Wu S, Li W (2012) CD-HIT: accelerated for clustering the next generation sequencing data. Bioinformatics 28:3150-3152

Hirshfeld FL (1976) Can X-ray data distinguish bonding effects from vibrational smearing? Acta Cryst A32:239-244

Hubbard SJ, Thornton JM (1993) NACCESS, Department of Biochemistry and Molecular Biology, University College London

Johnson TW, Gallego RA, Brooun A, Gehlhaar D, McTigue M (2018) Reviving B-Factors: retrospective normalized B-factor analysis of c-ros oncogene 1 receptor tyrosine kinase and anaplastic lymphoma kinase L1196M with crizotinib and lorlatinib. ACS Med Chem 9:878-883

Li W, Godzik A (2006) Cd-hit: a fast program for clustering and comparing large sets of protein or nucleotide sequences. Bioinformatics 22:1658-1659
Longhi S, Czjzek M, Cambillau C (1998) Messages from ultrahigh resolution crystal structures. Curr Opin Struct Biol 8:730-737

Parois P, Arnold J, Cooper R (2018) An enhanced set of displacement parameter restraints in CRYSTALS. J Appl Cryst 51:1059-1068

Reetz MT, Carballeira JD, Vogel A (2006) Iterative saturation mutagenesis on the basis of $\mathrm{B}$ factors as a strategy for increasing protein thermostability. Angew Chem Int Ed Eng 45:7745-7751

Schmidt A, Lamzin VS (2002) Veni, vidi, vici - atomic resolution unravelling the mysteries of protein function. Curr Opin Struct Biol 12:698-703

Schmidt A, Lamzin VS (2010) Internal motion in protein crystal structures. Protein Sci 19:944-953

Schmidt A, Teeter M, Weckert E, Lamzin VS (2011) Crystal structure of small protein crambin at $0.48 \AA$ resolution. Acta Cryst F67:424-428

Slater JC (1968) Quantum Theory of Matter. New York: McGraw-Hill Book Company

Sun Z, Liu Q, Qu G, Feng Y, Reetz MT (2019) Utility of B-factors in protein science: interpreting rigidity, flexibility, and internal motion and engineering. Chem Rev 119:1626-1665

Thorn A, Dittrich B, Sheldrick GM (2012) Enhanced rigid-bond restraints. Acta Cryst A68:448-451

Tronrud DE (1996) Knowledge-based B-factor restraints for the refinement of proteins. J Appl Cryst 29:100-104

Publisher's Note Springer Nature remains neutral with regard to jurisdictional claims in published maps and institutional affiliations. 